Proc. 15th Int. Conference on Defects Recognition, Imaging and Physics in Semiconductors, Warsaw, Poland 2013

\title{
Investigation on the Mechanisms of Nitrogen Shallow Implantation Influence on Trap Properties of $\mathrm{SiO}_{2} / n$-Type $4 \mathrm{H}$-SiC Interface
}

\author{
K. KRÓL ${ }^{a, b, *}$, M. SOCHACKI ${ }^{a}$ AND J. SzMIDT ${ }^{a}$ \\ ${ }^{a}$ Institute of Micro- and Optoelectronics, Warsaw University of Technology, Koszykowa 75, 00-662 Warszawa, Poland \\ ${ }^{b}$ Tele- and Radio Research Institute, Ratuszowa 11, 03-450 Warszawa, Poland
}

\begin{abstract}
Silicon carbide $(\mathrm{SiC})$ is the only wide-bandgap semiconductor capable of forming native dielectric layer of $\mathrm{SiO}_{2}$ by thermal oxidation. This unique property of $\mathrm{SiC}$ combined with its high thermal conductivity and high critical field makes this semiconductor material suitable for high power electronic devices. Unfortunately, the state-of-the art technology does not use the full benefits of the material, especially in the case of MOSFET transistors. This is caused by insufficient electrical parameters of $\mathrm{SiO}_{2} / \mathrm{SiC}$ interface. Two-component structure of the material and its high density result in high level of interface traps reducing the surface mobility and thus increasing series resistance of the device. One of the proposed methods of reducing the trap density in SiC MOS structure is a shallow nitrogen implantation prior to oxidation. This technique is based on the observation that introducing nitrogen into the $\mathrm{SiO}_{2} / \mathrm{SiC}$ system results in significant reduction of trap states density and increase of the channel effective mobility. The shallow implantation technique has been reported to be as much effective as nitric oxide annealing which is one of the most effective techniques for oxide quality improvement in case of SiC. Unlike the diffusion based techniques, like postoxidation annealing, implantation of the nitrogen prior oxidation has the possibility of nitrogen concentration control near the oxide interface during oxidation process itself. This property is important since it was shown that the improvement degree is directly proportional to amount of nitrogen built in the vicinity of $\mathrm{SiO}_{2} / \mathrm{SiC}$ interface during oxidation. However, previous investigations about this technique were inconclusive about the influence of implantation parameters and process conditions on observed effects. Both improvement and deterioration of interface quality was observed by different researchers. This behavior was never explained clearly. The primary objective of this research is to analyze the impact of implantation conditions on electrical properties of $\mathrm{SiO}_{2} / \mathrm{SiC}$ MOS structure. This analysis is used to evaluate a hypothetical description of physical phenomena during oxidation of shallowly implanted substrates.
\end{abstract}

DOI: 10.12693/APhysPolA.125.1033

PACS: 73.40.Qv, 68.55.ag, 73.40.Qv, 72.20.-i, 72.25.-b

\section{Introduction}

The demand for a new generation of power electronic devices results in increasing interest in wide-bandgap semiconductors. Therefore an extensive research effort has gone into the development of $\mathrm{SiC}$ power MOSFET having a superior performance to silicon devices. Silicon carbide has several advantages in comparison with other $\mathrm{A}^{\mathrm{IV}}-\mathrm{B}^{\mathrm{IV}}$ compounds such as chemical stability, high thermal conductivity, and high saturated electron velocity [1]. This material is capable of forming high quality native dielectric layer by thermal oxidation which is unique property of $\mathrm{SiC}$ making it preferable for manufacturing of high-power, high-temperature and high-frequency devices [2].

However, the complex chemical structure of the material in comparison with silicon and a multi-stage oxidation reaction promotes the formation of different impurities, mostly originating from carbon impurities in the form of carbon dimers [3], graphitic microcrystals [4] in

*corresponding author; e-mail: kkrol@stud.elka.pw.edu.pl conjunction with the occurrence of interstitial atoms of carbon and silicon [5]. All of these impurities have a significant contribution to the estimated trap density.

Another important source of trap states are bond length mismatch between silicon oxide and crystalline silicon carbide [6]. It results in significant stress at semiconductor/oxide interface and large amount of broken bonds.

A simple oxidation procedure leads to the formation of dielectric layer having large interface trap density effectively reducing the electron surface mobility. An extensive research effort has gone into improving the quality of the thermal oxide. It was shown that trap density can be decreased by an application of nitrogen ambient annealing [7-9]. The most significant trap reduction was achieved by application of $\mathrm{NO}$ annealing $[8,9]$.

More detailed investigation by means of secondary ion mass spectroscopy (SIMS) and X-ray photoelectron spectroscopy (XPS) shows that nitrogen-enhanced trap density reduction is proportional to nitrogen concentration at the vicinity of the interface region $[10,11]$.

Unfortunately, despite the continuous improvement in oxide quality, the $\mathrm{SiO}_{2} / \mathrm{SiC}$ interface is far from perfect one. This is partly due to the fact that nitrogen concentration at the interface region depends on the diffusion 
process through the growing dielectric layer. The diffusion process at given thermodynamic conditions shows a concentration limit due to the increasing nitrogen concentration near the interface region and a reduction of diffusion effectiveness.

To solve this problem several research groups investigated the incorporation of nitrogen at the interface region by application of shallow ion implantation prior to thermal oxidation [12-14]. This method helps to improve the surface mobility by more than one order of magnitude.

However, the published results were inconsistent on the measured trap density. Poggi et al. [13] reported a significant increase of trap density at energies localized near the $4 \mathrm{H}-\mathrm{SiC}$ conduction band as a function of increasing implantation dose. However, the electron mobility measured for MOSFET transistors using the highest dose implantation was the best among all characterized samples. This was explained by reduction of the near interface traps density.

A completely different behavior of trap density was observed by Okamoto et al. [14]. This group observed the decrease of trap density within energy range near the conduction band with increasing dose of implanted nitrogen. Similar results were reported by Dhar et al. [15]. Gurfinkel et al. [16] reported no significant change in carrier mobility caused by nitrogen implantation and MOSFETs performance was improved by subsequent NO annealing only. The primary objective of this research is a contribution to clarifying the mechanisms responsible for such different behavior of the observed electrical properties of thermally oxidized $\mathrm{SiC}$ with implanted nitrogen.

\section{Experimental}

In this work, five $n$-type $4 \mathrm{H}-\mathrm{SiC}$ (0001) samples manufactured on the device-grade nitrogen doped substrates purchased from SiCrystal AG were used with $10 \mu \mathrm{m}$ epitaxial layer doped with nitrogen $\left(1 \times 10^{16} \mathrm{~cm}^{-3}\right)$. The epitaxial layer was fabricated at Institute of Electronic Materials Technology. Sample \#1 was the reference and it was not subjected to implantation. Remaining samples were implanted through the stopping mask to obtain a fast decaying profile of implanted nitrogen as a function of depth in sample substrate. In all cases nitrogen concentration at $50 \mathrm{~nm}$ depth was smaller than $1 \%$ of surface nitrogen concentration. Each sample was implanted with different dose of $\mathrm{N}_{2}$ to obtain wide cross-section of surface concentration of nitrogen. Next, all samples were oxidized in dry $\mathrm{O}_{2}$ at $1200^{\circ} \mathrm{C}$ to obtain a required thickness of oxide layer.

Implantation dose and nitrogen implantation at the depth corresponding to the position of interface of oxidized sample are shown in Table I. No post-implantation annealing was performed. It was made deliberately to prevent the implanted nitrogen from building into the substrate crystal structure.

The aim of nitrogen implantation in this experiment was to introduce the nitrogen particles into the region
The basic parameters of samples.

TABLE I

\begin{tabular}{c|c|c|c}
\hline $\begin{array}{c}\text { Sample } \\
\text { No. }\end{array}$ & $\begin{array}{c}\text { Implantation } \\
\text { dose } \\
{\left[\mathrm{cm}^{-2}\right]}\end{array}$ & $\begin{array}{c}\text { Nitrogen } \\
\text { interface } \\
\text { concentration } \\
{\left[\mathrm{cm}^{-3}\right]}\end{array}$ & $\begin{array}{c}\text { Thermal } \\
\text { oxide } \\
\text { thickness } \\
{[\mathrm{nm}]}\end{array}$ \\
\hline$\# 1$ & - & - & 77.1 \\
$\# 2$ & $\approx 1.5 \times 10^{13}$ & $3.3 \times 10^{16}$ & 49.5 \\
$\# 3$ & $\approx 1.5 \times 10^{14}$ & $1.0 \times 10^{17}$ & 33.7 \\
$\# 4$ & $\approx 1.5 \times 10^{15}$ & $3.4 \times 10^{18}$ & 50.4 \\
$\# 5$ & $\approx 1.5 \times 10^{16}$ & $2.5 \times 10^{19}$ & 35.8
\end{tabular}

where $\mathrm{SiO}_{2} / \mathrm{SiC}$ interface was expected to be localized after oxidation and to keep these particles mobile at elevated temperatures allowing them to efficiently passivate carbon defects that are created during thermal oxidation. The subsequent measurements of $1 / C^{2}$ characteristics of MOS structures confirmed that this goal was achieved.

Next, the circular MOS capacitors with $\mathrm{Al}$ gate area of $1.8 \times 10^{-4} \mathrm{~cm}^{2}$ were fabricated for electrical characterization and the backside ohmic contact was formed. More specific details of technology used can be found in [17]. The influence of implantation parameters on basic electrical parameters was investigated by $\mathrm{HF} C-V$ measurements using Keithley 4200 Semiconductor Characterization System. A trap density $\left(D_{\text {it }}\right)$ profile near the conduction band was calculated using HF method.

Since the relationship between the extracted parameters and implantation damage is expected, the amount of vacancies (both silicon and carbon) was simulated with SRIM software to determine the theoretical level of substrate damage and its depth profile. The damage results were normalized to silicon carbide atomic density to obtain the final depth profiles of percentage damage. The physical basis of damage calculation can be found in [18].

\section{Results and discussion}

Trap density profile extracted from $C-V$ measurements for all samples used in the experiment is shown in Fig. 1. The observed effects depend on the locations of the investigated traps in $4 \mathrm{H}-\mathrm{SiC}$ bandgap.

The traps located near the conduction band edge (approximately $\approx 3.2 \mathrm{eV}$ for $4 \mathrm{H}-\mathrm{SiC}$ ) are the most critical due to the highest concentration level. As shown in Fig. 1, low dose of implantation results in the reduction of these traps comparing to the unimplanted sample. The lowest trap density is related to the lowest implantation dose despite the fact that subsurface concentration of the implanted nitrogen for sample $\# 2$ is comparable to the epitaxial layer doping. The significant improvement was only observed for sample $\# 2$ and $\# 3$. However, no significant change of the trap density was observed deeper in the bandgap (below $3 \mathrm{eV}$ ).

Sample \# 4 with higher implantation dose shows different behavior. The implantation dose results in the lack of improvement of shallow trap density level comparing to the reference (sample \#1), but it leads to a significant 


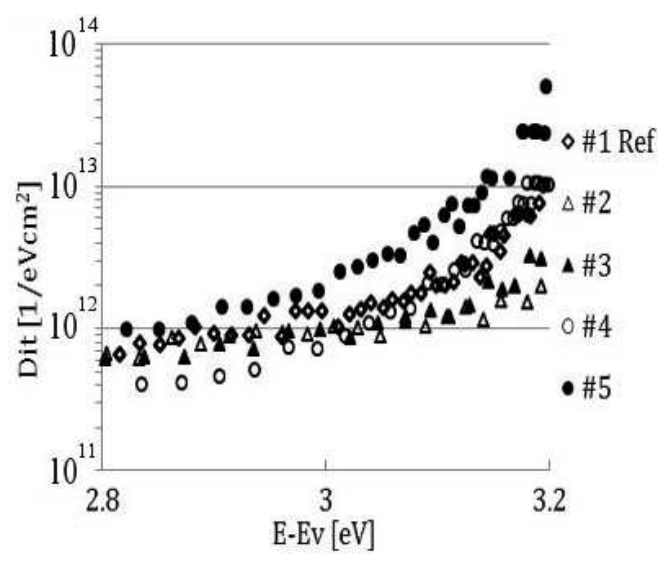

Fig. 1. Trap density profile as a function of location in $4 \mathrm{H}-\mathrm{SiC}$ bandgap.

decrease of trap density deeper in the bandgap. This effect was also observed for $\mathrm{NO}$ or $\mathrm{N}_{2} \mathrm{O}$ annealing [19] and it has been explained by creation of $\mathrm{C}=\mathrm{N}$ bonds since according to thermodynamic simulation [20] the trap levels at midgap are caused mostly due to the existence of carbon impurities.

Therefore, it was assumed in this work that the moderate nitrogen implantation dose is enough to efficiently neutralize carbon impurities. This experiment is fully consistent with the result presented by Dhar et al. [16] who used similar nitrogen concentration as in sample \#4.

Finally, one can observe a significant increase of trap density for sample \#5 within whole bandgap energy range (especially for traps located near the conduction band). The following hypothesis was proposed to explain the results. The traps located close to the conduction band correspond to broken bonds and vacancies that exist due to large difference in bond length between $\mathrm{SiO}_{2}$ and $\mathrm{SiC}$. In fact, recent density functional (DFT) theory simulations have shown that these types of defects can be responsible for introducing trap levels very close to conduction band and are not efficiently passivated by the nitrogen [21].

Based on this assumption, the improvement of the trap density near the conduction band for the lowest implantation dose can be explained by reduction of the interface stress by residual nitrogen implanted atoms. As a result, this type of defect creation is decreased due to smaller force gradient. These doses (sample \#2 and \#3) are still too small for efficient passivation of carbon impurities because the concentration of implanted nitrogen is comparable to nitrogen concentration in the epitaxial layer. Thus no improvement of traps density can be observed in the midgap.

The level of damage increases with the increasing dose of implantation. The accumulation of post-implantation damage results in the increase of traps density near the conduction band and the lowering of carbon-originated traps located deeper in $4 \mathrm{H}-\mathrm{SiC}$ bandgap (due to increas- ing nitrogen concentration enhancing the passivation effect described earlier). At the highest dose, the damage is so large so the traps generated by this effect are higher with comparison to the unimplanted sample.

The sample \#5 shows rapid increase of the trap density at energy higher than approximately $2.9 \mathrm{eV}$ but the trap density is comparable to the reference deeper in the bandgap. Such behavior is an expected phenomenon, since structural defects caused by implantation may create traps at energy located mostly close to the conduction band edge (calculated energy levels for Si broken bonds are $E_{\mathrm{V}}+3.2 \mathrm{eV}, E_{\mathrm{V}}+3.0 \mathrm{eV}$, and $E_{\mathrm{V}}+2.5 \mathrm{eV}$ [21]). Our results of the Raman spectroscopy measurements have confirmed proportional dependence between crystal damage and trap density near the conduction band [17].
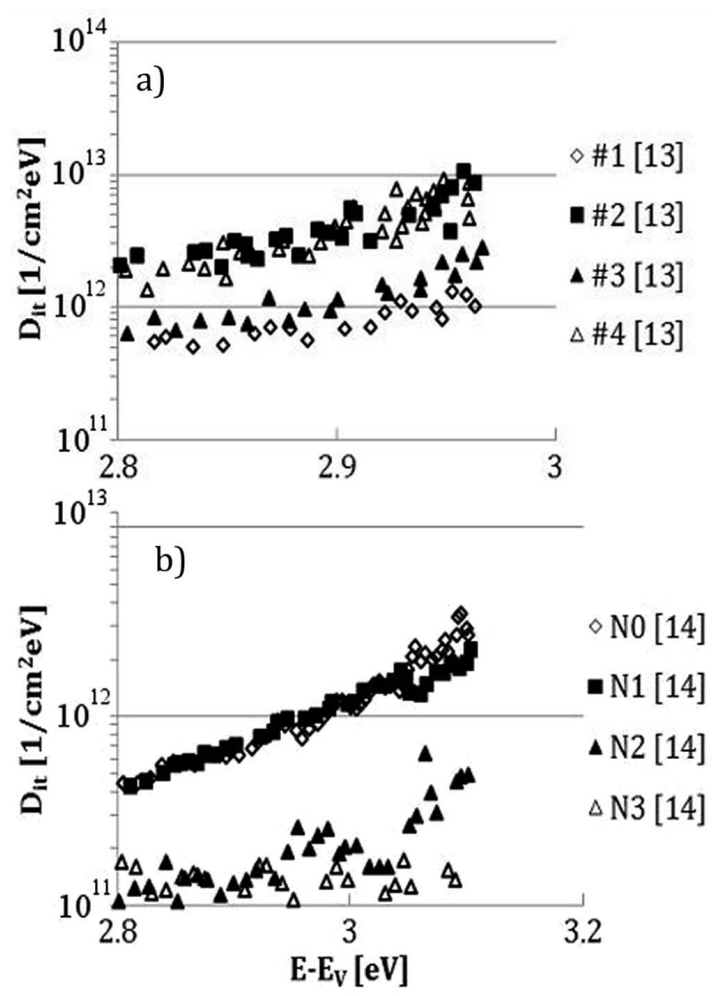

Fig. 2. Analyzed $D_{\text {it }}$ energy profiles for energy close to conduction band edge. (a) Data from [13]. (b) Data from [14].

To validate our hypothesis we have used a larger set of data gathered by different researchers. Figure 2 shows the $D_{\text {it }}$ profiles and Table II contains the implantation parameters. Poggi et al. [13] performed an implantation experiment using 3 runs of implantation and utilizing a variety of different energies and doses (see Table II). This resulted in a wide variety of nitrogen concentration profile. On the other hand, Okamoto et al. [14] has applied low energy and low implantation dose using the same set of energy and different doses from a relatively small range (Table III). All researchers used fast decaying as a function of depth implantation profiles similar to that used 
by authors (except sample \#4 from [13] where double implantation resulted in an extended profile).

TABLE II

Implantation process parameters based on [13].

\begin{tabular}{c|c|c|c}
\hline \hline No. & $\begin{array}{c}N \text { concentration } \\
{\left[\mathrm{cm}^{-3}\right]}\end{array}$ & $\begin{array}{c}\text { Dose } \\
{\left[\mathrm{cm}^{-2}\right]}\end{array}$ & $\begin{array}{c}\text { Energy } \\
{[\mathrm{keV}]}\end{array}$ \\
\hline$\# 1$ & - & - & - \\
$\# 2$ & $5 \times 10^{18}$ & $1 \times 10^{13}$ & 10 \\
$\# 3$ & $-{ }^{* *}$ & $1 \times 10^{15}$ & 2.5 \\
$\# 4^{*}$ & $1 \times 10^{19}$ & $5 \times 10^{14}$ & 2.5 \\
& & $5 \times 10^{14}$ & 5 \\
\hline
\end{tabular}

* extremally shallow implantation;

** double implantation

TABLE III

Implantation process parameters based on [14]

\begin{tabular}{c|c|c|c}
\hline \hline No. & $\begin{array}{c}N \text { concentration } \\
{\left[\mathrm{cm}^{-3}\right]}\end{array}$ & $\begin{array}{c}\text { Dose } \\
{\left[\mathrm{cm}^{-2}\right]}\end{array}$ & $\begin{array}{c}\text { Energy } \\
{[\mathrm{keV}]}\end{array}$ \\
\hline N0 & - & - & - \\
N1 & $3 \times 10^{15}$ & $1 \times 10^{12}$ & 15 \\
N2 & $1.5 \times 10^{16}$ & $5 \times 10^{12}$ & 15 \\
N3 & $3 \times 10^{16}$ & $1 \times 10^{13}$ & 15
\end{tabular}

In case of $D_{\text {it }}$ profiles obtained in [13] no correlation can be observed between implantation dose or energy and resulting trap density. Nevertheless by using low energy implantation with constant energy, Okamoto et al. [14] resulted in decrease of $D_{\text {it }}$ as a function of dose. This result is in opposite to findings reported by our team here and in [17]. However all those results support our hypothesis.

One can observe that in case of [13] implantation processes that introduce considerable amount of damage either by high energy (sample \#2 [13]) or by increased dose (double implanted sample \#4 [13]) a deterioration of trap density close to conduction band can be observed. The third process with moderate parameters has only slight influence on trap density in this region. The observation of Okamoto et al. [14] was made at one order of magnitude smaller dose and much thicker oxide. This resulted in consumption of highly damaged substrate layer and the damage near the interface. It leads to improvement of trap density near the conduction band. It is in accordance with our result since the improvement was made at nitrogen concentration level near the interface comparable to those of the substrate (as in our studies). Therefore, it cannot be connected to carbon defect passivation. To check our hypothesis we have made simulations of all described implantation procedures described in this paper calculating a percentage damage to the substrate crystal and its distribution. Figure 3 shows the obtained results.

Regardless of sample, the severe deterioration of trap density near the conduction band is always obtained for samples with process parameters resulting in high damage to the crystal near the interface region. If damage

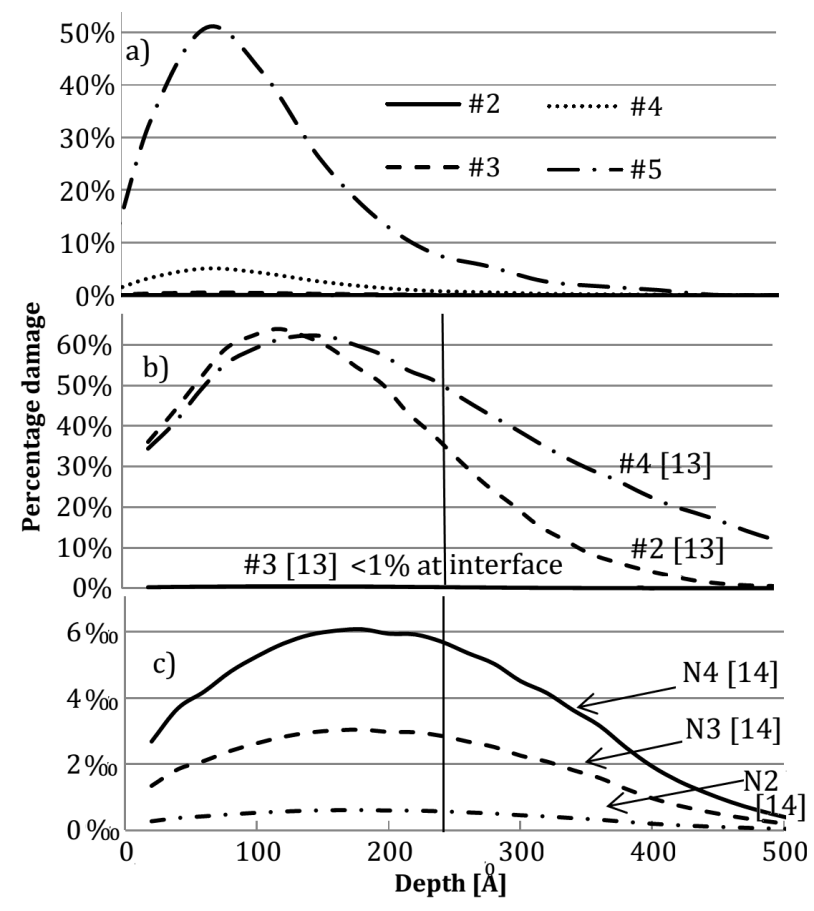

Fig. 3. Percentage damage calculations of samples used in described experiment (a) and known from Refs. [13] (b) and [14] (c). Vertical line marks the interface.

level near the interface is higher than roughly $10 \%$ of all $\mathrm{SiC}$ substrate atoms no improvement can be obtained. Regardless of parameters, one can observe the decrease of this trap density providing sufficiently small damage to the crystal (smaller than $\approx 5 \%$ ) even if the concentration of nitrogen is small (comparable to substrate concentration). These results support the hypothesis that defect centers responsible for high level of trap density close to the conduction band in $4 \mathrm{H}-\mathrm{SiC}$ are related rather to structural defects (Si broken bonds) than to carbon related defects.

\section{Conclusions}

We have proposed a hypothesis which endeavours to explain the electrical behavior of traps in such a way that U-shaped trap profile maximum after thermal oxidation of nitrogen implanted samples is defined more by the interface structural defects than carbon impurities. This mechanism is applicable both to our own studies and the results in the literature. Moreover, the mechanism is also consistent with the results of oxidation and/or annealing in nitrogen containing atmospheres and supported by theoretical DFT simulation results.

\section{Acknowledgments}

This work was supported by Polish National Science Center under project no. N N515 498340 "Influence of 
shallow ion implantation of $4 \mathrm{H}-\mathrm{SiC}$ on electro-physical properties of MOS structure" and project no. UMO$-2011 / 01 / \mathrm{D} / \mathrm{ST} 7 / 00640$ "Ultra-shallow plasma ion implantation for technology of advanced MOS/MOSFET structures fabricated on silicon and silicon carbide characterization of phenomenon, attempts for technological optimization". This work was co-financed by the European Union under the European Social Fund.

\section{References}

[1] A M. Huang, N. Goldsman, C. Chang, I. Mayergoyz, J.M. McGarrity, D. Woolard, J. Appl. Phys. 84, 2065 (1998).

[2] C.M.M. Zetterling, B.L. Weiss, Process Technology for Silicon Carbide Devices, Institution of Electrical Engineers, Stevenge 2002.

[3] V.V. Afanasev, M. Bassler, G. Pensl, M. Schulz, Phys. Status Solidi A, Appl. Res. 162, 321 (1997).

[4] P. Borowicz, T. Gutt, T. Małachowski, M. Łatek, Przeglad Elektrotechniczny 11a, 123 (2010).

[5] Y. Hijikata, H. Yaguchi, S. Yoshida, Appl. Phys. Expr. 2, 021203 (2009).

[6] J. Bernhardt, J. Schardt, U. Starke, K. Heinz, Appl. Phys. Lett. 74, 1084 (1999).

[7] D. Okamoto, H. Yano, Y. Oshiro, T. Hatayama, Y. Uraoka, T. Fuyuki, Appl. Phys. Expr. 2, 021201 (2009).

[8] R. Kosugi, T. Umeda, Y. Sakuma, Appl. Phys. Lett. 99, 182111 (2011).

[9] G.V. Soares, I.J.R. Baumvol, L. Hold, F. Kong, J. Han, S. Dimitrijev, C. Radtke, F.C. Stedile, Appl. Phys. Lett. 91, 041906 (2007).

[10] K. McDonald, R.A. Weller, S.T. Pantelides, L.C. Feldman, G.Y. Chung, C. Tin, J.R. Williams, J. Appl. Phys. 93, 2719 (2003).
[11] J. Rozen, S. Dhar, M.E. Zvanut, J.R. Williams, L.C. Feldman, J. Appl. Phys. 105, 124506 (2009).

[12] F. Moscatelli, A. Poggi, S. Solmi, R. Nipoti, IEEE Trans. Electron Dev. 55, 961 (2008).

[13] A. Poggi, F. Moscatelli, S. Solmi, R. Nipoti, IEEE Trans. Electron Dev. 55, 2021 (2008).

[14] D. Okamoto, H. Yano, T. Hatayama, T. Fuyuki, Mater. Sci. Forum 645-648, 495 (2009).

[15] S. Dhar, S. Ryu, A.K. Agarwal, IEEE Trans. Electron Dev. 57, 1195 (2010).

[16] M. Gurfinkel, S. Potbhare, H.D. Xiong, J.S. Suehle, Yoram Shapira, A.J. Lelis, D. Habersat, N. Goldsman, J. Appl. Phys. 105, 084511 (2009).

[17] K. Król, M. Sochacki, M. Turek, J. Zuk, H.M. Przewlocki, T. Gutt, P. Borowicz, M. Guziewicz, J. Szuber, M. Kwoka, P. Koscielniak, J. Szmidt, Mater. Sci. Forum 740-742, 733 (2013).

[18] J.F. Ziegler, J.P. Biersack, M.D. Ziegler, SRIM The Stopping and Range of Ions in Matter, James Ziegler, Annapolis 2008.

[19] P. Jamet, S. Dimitrijev, P. Tanner, J. Appl. Phys. 90, 5058 (2001).

[20] P. Deák, J. Knaup, C. Thill, T. Frauenheim, T. Hornos, A. Gali, J. Phys. D, Appl. Phys. 41, 049801 (2008).

[21] S. Salemi, N. Goldsman, A. Akturk, A. Lelis, in: Proc. Int. Conf. on Simulation of Semiconductor Process and Devices SISPAD 2012, Denver (USA), IEEE, Piscataway 2012, p. 121. 